OPEN ACCESS

Edited by:

Ruiwen Zhang,

University of Houston, United States

Reviewed by:

Sachchida Nand Rai, University of Allahabad, India

Saumitra Sen Singh,

Banaras Hindu University, India Hareram Birla,

Banaras Hindu University, India

${ }^{*}$ Correspondence:

Yunling Zhang

yunlingzhang2004@126.com

Specialty section:

This article was submitted to

Ethnopharmacology,

a section of the journal

Frontiers in Pharmacology

Received: 10 July 2020

Accepted: 09 October 2020

Published: 23 November 2020

Citation:

Du W, Liang $X$, Wang S, Lee $P$ and

Zhang Y (2020) The Underlying Mechanism of Paeonia lactiflora Pall. in Parkinson's Disease Based on a

Network Pharmacology Approach.

Front. Pharmacol. 11:581984.

doi: 10.3389/fphar.2020.581984

\section{The Underlying Mechanism of Paeonia lactiflora Pall. in Parkinson's Disease Based on a Network Pharmacology Approach}

\author{
Wanqing $\mathrm{Du}^{1,2}$, Xiao Liang $^{2}$, Shanze Wang ${ }^{3}$, Philip Lee ${ }^{1}$ and Yunling Zhang ${ }^{2,4 *}$ \\ ${ }^{1}$ Graduate School, Beijing University of Chinese Medicine, Beijing, China, ${ }^{2}$ Department of Neurology, Xiyuan Hospital, China \\ Academy of Chinese Medical Sciences, Beijing, China, ${ }^{3}$ Dongzhimen Hospital, Beijing University of Chinese Medicine, Beijing, \\ China, ${ }^{4}$ Dongfang Hospital, Beijing University of Chinese Medicine, Beijing, China
}

Background: Parkinson's disease (PD) is the second most common neurodegenerative disease worldwide, yet as of currently, there is no disease-modifying therapy that could delay its progression. Paeonia lactiflora Pall. is the most frequently used herb in formulas for PD in Traditional Chinese Medicine and also a potential neuroprotective agent for neurodegenerative diseases, while its mechanisms remain poorly understood. In this study, we aim to explore the underlying mechanism of $P$. lactiflora in treating PD utilizing a network pharmacology approach.

Methods: The protein targets of $P$. lactiflora ingredients and PD were first obtained from several databases. To clarify the key targets, a Protein-Protein-Interaction (PPI) network was constructed and analyzed on the String database, and then enrichment analysis was performed by the Metascape platform to determine the main Gene Ontology biological processes and Kyoto Encyclopedia of Genes and Genomes pathways. Finally, the Ingredient-Target-Pathway (I-T-P) network was constructed and analyzed by Cytoscape software.

Results: Six active ingredients of $P$. lactiflora (kaempferol, B-sitosterol, betulinic acid, palbinone, paeoniflorin and (+)-catechin) as well as six core targets strongly related to $P D$ treatment [AKT1, interleukin-6, CAT, Tumor necrosis factor (TNF), CASP3, and PTGS2] were identified. The main pathways were shown to involve neuroactive ligand-receptor interaction, Calcium signaling pathway, PI3-Akt signaling pathway, TNF signaling pathway, and apoptosis signaling pathway. The main biological process included the regulation of neurotransmitter levels.

Conclusion: $P$. lactiflora may retard neurodegeneration by reducing neuroinflammation, inhibiting intrinsic and extrinsic apoptosis, and may improve motor and non-motor symptoms by regulating the levels of neurotransmitters. Our study has revealed the mechanism of $P$. lactiflora in the treatment of $P D$ and may contribute to novel drug development for PD.

Keywords: Parkinson's disease, Paeonia lactiflora Pall., network pharmacology, multi-target, Traditional Chinese Medicine, apoptosis 


\section{INTRODUCTION}

Parkinson's disease (PD) is becoming the fastest growing neurological disorder surpassing Alzheimer's disease (AD), with the number of patients expected to double to 14.2 million by 2040 (Dorsey and Bloem, 2018). The clinical features of PD are heterogeneous and complex, including typical motor symptoms such as bradykinesia, resting tremor, rigidity, and postural instability, as well as non-motor symptoms (NMS) such as hyposmia, sleep disorders, autonomic nervous dysfunction, and mental and cognitive disorders (Kalia and Lang, 2015). The objectives of treatment involve symptomatic treatment and disease-modifying therapy (de Bie et al., 2020). However, adverse events of symptomatic supplement with levodopa, such as dyskinesia, fluctuations, psychiatric symptoms are frequently observed in the advanced stage of the disease (National Institute for Health and Care Excellence, 2017), and drugs for NMS are quite limited (Seppi et al., 2019). At present, no disease-modifying therapy is available to delay the progression of the disease (Kalia and Lang, 2015), thus underlining the urgency to discover drugs to change the course of the disease and improve symptoms. In view of the complicated pathological mechanism of $\mathrm{PD}$, including abnormal aggregation of $\alpha$-synuclein, inflammation, oxidative stress, and apoptosis, novel drugs may need to target multiple pathways at the same time (Elkouzi et al., 2019).

For this reason, medicinal plants with multiple ingredients have received widespread attention for PD neuroprotection. A previous research has already summarized more than 50 types of plants against MPTP induced neurotoxicity (Abushouk et al., 2017), including Mucuna pruriens seeds and P. lactiflora roots. Many of these herbs were discovered in traditional medicine practice, and now verified by modern experiments to play a role in various pathological process of PD.

In China, where Traditional Chinese medicine (TCM) accounts for an incredible $28.55 \%$ of its total pharmaceutical industry (WHO, 2019), P. lactiflora is reported to be the most frequently used herb in the treatment of PD (Sun and Peng, 2017). The dried root of Paeonia lactiflora Pall., also known as Paeoniae Radix Alba, or Bai shao in Chinese, has been applied in TCM for 2000 years since first recorded in the Prescriptions for Fifty-two Aliments (Yan, 2005) and is still widely used to improve the symptoms of PD patients. The reason behind this choice should be attributed to TCM theory. Traditionally, motor symptoms like tremor and rigidity are ascribed to the dysfunction of the "Liver system," and P. lactiflora could "nourish and tranquillize the liver system" to alleviate the symptoms (You, 2009). Literature suggests that P. lactiflora has crucial influence on the nervous and immune systems, and the therapeutic effects can be described as neuroprotective, antidepressant, sedative, analgesic, and anticonvulsant (Tan et al., 2020). According to the Pharmacopoeia of the People's Republic of China (2010), indications of $P$. lactiflora involve excessive sweating, abdominal pain, contracture of limbs, headache and dizziness, irregular menstruation, et al. (National Pharmacopoeia Commission, 2010). Diarrhea was reported as the most common adverse effect of $P$. lactiflora extracts (Feng et al.,
2019); hence it is sometimes used for constipation. Modern studies have found that the Total Glucosides of Paeony (TGP), a capsule form extracted from $P$. lactiflora, exerts neuroprotective effect in MPTP-induced PD mice by activating cAMP/PKA/ CREB on apoptosis pathway (Zheng et al., 2019), and paeoniflorin can reduce the neurotoxicity induced by glutamate in pheochromocytoma (PC12) cells (Sun et al., 2012). Furthermore, TGP capsule has a safe history of practice in the disease-modifying treatment of rheumatoid arthritis since being approved on market in China in 1998 (Luo et al., 2017), with anti-inflammatory and immunomodulatory effects (Zhang and Wei, 2020). Therefore, P. lactiflora may be a highly promising drug for PD.

Network pharmacology is a novel approach to analyze drug mechanisms, identify new targets, and expand new indications at a systematic level, which can comprehensively reflect the mechanism of drugs on disease networks, thus providing support for drug discovery for complex diseases and natural products (Hopkins, 2007; Kibble et al., 2015). Network pharmacology has become an effective method to predict the mechanism of TCM herbs and decoctions in the treatment of neurodegenerative diseases such as PD (Ke et al., 2016; Li et al., 2020). Therefore, this study intends to elucidate the underlying mechanism of $P$. lactiflora in the treatment of PD based on a network pharmacology approach from a holistic view.

\section{MATERIALS AND METHODS}

\section{Ingredients and Targets Screening of Paeonia lactiflora}

To obtain the active ingredients of $P$. lactiflora, an initial screening based on the absorption, distribution, metabolism, and excretion (ADME) properties [oral availability $(\mathrm{OB}) \geq$ $30 \%$ and drug-likeness $(\mathrm{DL}) \geq 0.18$ ], was conducted by the TCM Systems Pharmacology (TCMSP, http://tcmspw.com/ tcmsp.php) database (Ru et al., 2014). OB represents the proportion of oral medication absorbed into the circulation, and DL is a filter to exclude the non-drug-like molecules. These early-stage pharmacokinetic evaluations are of vital importance to reduce the probability of unsuccessful drug discovery (Wang and Urban, 2004). Since pathological and animal evidence have supported that PD may originate from the gut and spread to the central nervous system (Klingelhoefer and Reichmann, 2015), it is speculated that drugs may function outside the brain, so the blood-brain barrier permeability was not used as a criterion for drug screening. The names and structure formulas of compounds were verified using PubChem (https:// pubchem.ncbi.nlm.nih.gov/) and the concentration of active components in this study were retrieved from literature.

Next, the known targets of these ingredients were collected from TCMSP and Swiss Target Prediction (http://www. swisstargetprediction.ch/) database (Gfeller et al., 2014). After screening, protein targets were standardized as gene symbol in Uniprot database (UniProt Consortium, 2019). 
TABLE 1 | Chemical properties of Paeonia lactiflora Pall. active ingredients.

Code Molecular name InCHI key

\section{InCHI key}

MOLID

Molecula
formula

\section{Structure} formula

Structure
formula

MW
$(\mathrm{g} / \mathrm{mol})$

OB (\%)

DL

480

PLP2 Palbinone

PLP3 kaempferol

PLP4 (+)-catechin

PLP5 betulinic acid (mairin)

PLP6 beta-sitosterol

PLP7 paeoniflorgenone

PLP8 albiflorin_qt

PLP9 benzoyl paeoniflorin
YKRGDOXKVOZESV-WRJNSLSBSA-N

KIAKLFLISZCITK-PPAUHQMUSA-N

IYRMWMYZSQPJKC-UHFFFAOYSA-N

PFTAWBLQPZVEMU-DZGCQCFKSA-N

QGJZLNKBHJESQX-FZFNOLFKSA-N

KZJWDPNRJALLNS-VJSFXXLFSA-N

BANPEMKDTXIFRE-GHWWTTSJSA-N

WSVOZDIRZKFUCH-UNTBTUBKSA-N

KHRHASRIMPQOPU-JEWJNOKWSA-N
MOL001924

MOL001919

$\mathrm{C}_{22} \mathrm{H}_{30} \mathrm{O}_{4}$

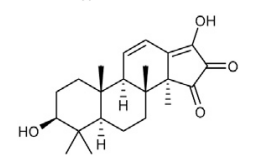

MOL000422

$\mathrm{C}_{15} \mathrm{H}_{10} \mathrm{O}_{6}$

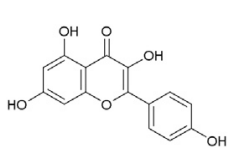

MOL000492

$\mathrm{C}_{15} \mathrm{H}_{14} \mathrm{O}_{6}$

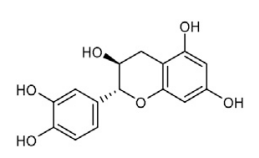

MOL000211

$\mathrm{C}_{30} \mathrm{H}_{48} \mathrm{O}_{3}$

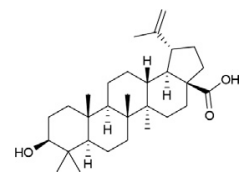

MOL000358, MOL00359

$\mathrm{C}_{29} \mathrm{H}_{50} \mathrm{O}$

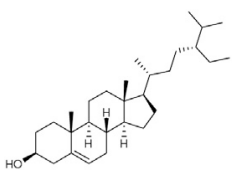

MOL001918

$\mathrm{C}_{17} \mathrm{H}_{18} \mathrm{O}_{6}$

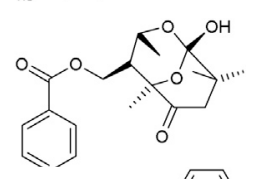

MOL001928

$\mathrm{C}_{23} \mathrm{H}_{28} \mathrm{O}_{11}$

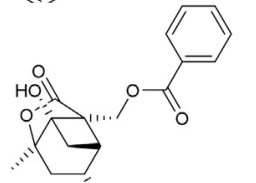

MOL001930 $\begin{array}{llll}480.5 & 53.87 & 0.79 & 26.27\end{array}\left(\begin{array}{ll}14 & \end{array}\right.$

358.5

43.56

0.53

1.66 (Kadota et al., 1993)

88.24

41.88

0.24

Identified (Shu et al., 2014)

290.27

$54.83 \quad 0.24 \quad 0.37$ (Liu et al., 2015)

$55.38 \quad 0.78$

N/A

414.7

36.91

0.75 Identifie d (Wu et al., 2020)

318.35

87.59

0.37 identified (Wu et al., 2020)

318.35

66.64

0.339 .83 (Liu et al., 2015)

(Continued on following page) 


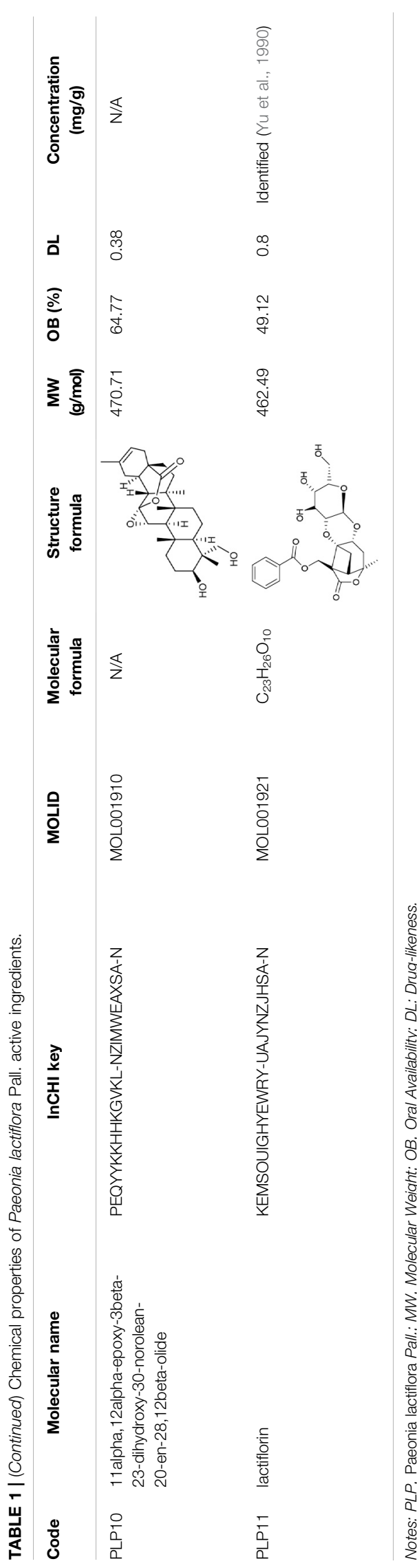

\section{Disease Targets Acquisition of Parkinson's Disease}

Using "PD" as the search term, we merged the obtained disease targets from three databases, OMIM (http://www.omim.org) (Amberger et al., 2015), Disgenet (https://www.disgenet.org/) (Piñero et al., 2017), and Drugbank (https://www.drugbank.ca) (Wishart et al., 2018), followed by deduplication.

\section{Protein-Protein-Interaction Network Analysis}

We acquired the common targets of the drug and disease by intersection and then constructed a Protein-Protein-Interaction (PPI) network on the STRING11.0 database (https://string-db. org) (Szklarczyk et al., 2017). Set the biological species as "Homo sapiens" and hide the unconnected nodes. In addition, the network topology parameters were visualized and analyzed by CytoScape3.7.2 (Shannon et al., 2003) to identify the core targets. The Degree value represents the number of nodes connected by a node, so the higher the Degree of a node, the more important it is in the network. If there were too many targets, a PPI core network would be extracted twice with nodes whose Degree, Betweenness centrality (BC), and Closeness centrality (CC) values were all greater than the median.

\section{Gene Ontology Biological Process and Kyoto Encyclopedia of Genes and Genomes Enrichment Analysis}

To illustrate the targets on a systematic level, the Gene Ontology Biological Process (GOBP) and Kyoto Encyclopedia of Genes and Genomes (KEGG) pathway enrichment analysis were carried out on the Metascape platform (http://metascape.org/gp/index.html). Metascape platform has integrated over 40 knowledgebases with monthly data updates and friendly interactive interface (Zhou et al., 2019). Items with a $p$-value $<0.01$, count $\geq 3$, and enrichment factor $>1.5$ were collected and grouped into clusters based on their similarities automatically by the platform.

\section{Ingredient-Target-Pathway Network Analysis}

To investigate the interaction among the active ingredients, common targets and the key KEGG pathway, the IngredientTarget-Pathway (I-T-P) network of P. lactiflora for PD was constructed and visualized by CytoScape3.7.2. The main active components were judged according to the network topology parameters which was analyzed by its built-in network analyzer.

\section{RESULTS}

\section{Ingredients and Targets Screening of Paeonia lactiflora}

11 active ingredients were extracted from 85 ingredients of $P$. lactiflora by ADME screening (Table 1). After deleting the duplicate targets obtained by TCMSP database and Swiss 


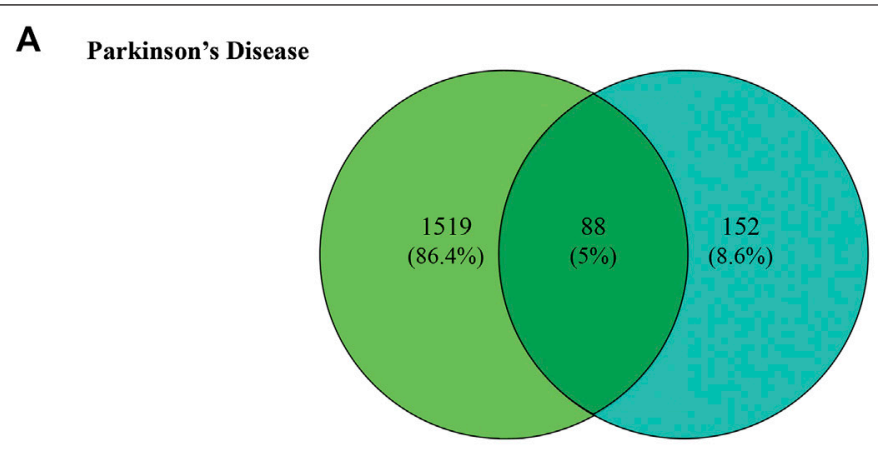

Paeonia lactiflora Pall.

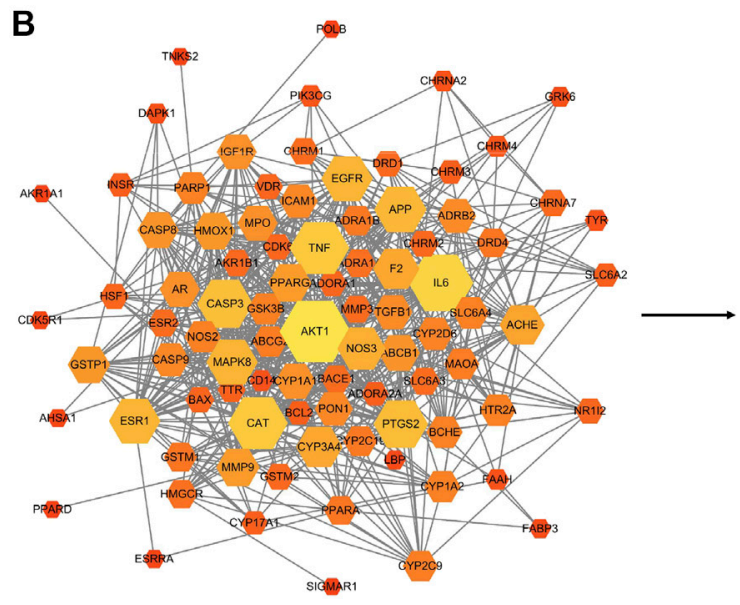

C

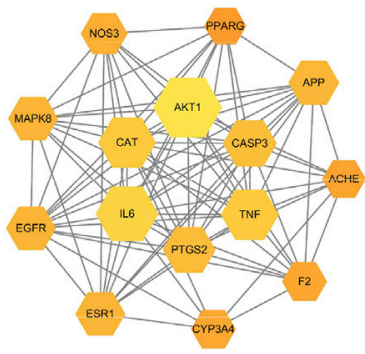

15 nodes, 86 edges

88 nodes, 688 edges

FIGURE 1 | (A) Common targets of Paeonia lactiflora Pall. and Parkinson's disease. (B) Protein-Protein-Interaction (PPI) network of putative targets. (C) PPI core network. The size and color of the node represent its Degree. Degree equals the number of nodes connected by a node. The larger and brighter the node is, the more important it is in the network.

Target Prediction, a total of 240 targets of $P$. lactiflora were collected.

\section{Disease Targets Acquisition of Parkinson's Disease}

523 targets from OMIM, 97 from Drugbank, and 1,063 from Disgenet database were integrated and finally 1607 PD targets were obtained.

\section{Protein-Protein-Interaction Network Analysis}

A Venn diagram was rendered to show the 88 common targets between $P$. lactiflora ingredients and PD (Figure 1A). In the String database, PPI network was established consisting of 88 nodes and 688 edges (Figure 1B). Based on the analysis of the topological characteristics of PPI network, AKT1 was the most important target in the network (Degree $=51, \mathrm{BC}=0.1374$, $\mathrm{CC}=0.7073$ ). To make it clear, PPI core network of 15 nodes and 86 edges was further extracted (Figure 1C). Consequently, top 6 targets, AKT1, interleukin-6 (IL-6), CAT, Tumor necrosis factor
(TNF), CASP3, and PTGS2, were predicted to be the key targets of $P$. lactiflora in the treatment of PD.

\section{Gene Ontology Biological Process and Kyoto Encyclopedia of Genes and Genomes Enrichment Analysis}

Through the enrichment analysis of 88 common targets on the Metascape platform, 1,436 GOBP items were collected and clustered by the platform, and top 20 clusters were selected and arranged according to the ascending order of $p$ value, as shown in Figure 2A. Results show that the main biological processes involved the response to toxic substances, regulation of neurotransmitter levels, apoptosis signaling pathway, neuron death, reactive oxygen species (ROS) metabolic process, and so on.

After removing pathways of other diseases, the top 20 KEGG pathways with the lowest $\mathrm{p}$ value and $\mathrm{PD}$ signaling pathway were shown in Figure 2B. The main pathways involved were neuroactive ligand-receptor interaction, Calcium signaling pathway, P450 Drug metabolism-cytochrome P450, TNF signaling pathway, IL-17 signaling pathway, HIF-1 signaling 

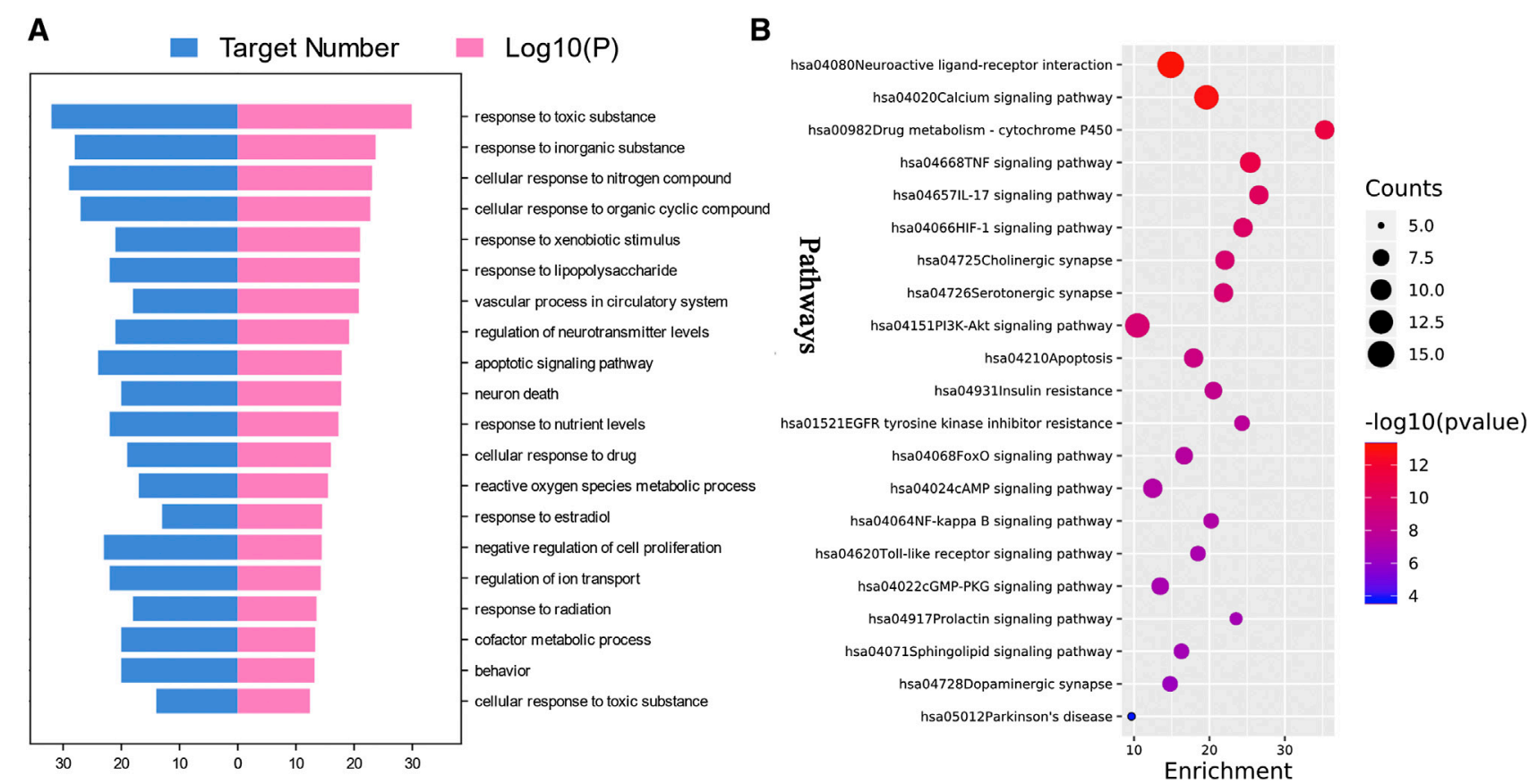

FIGURE 2 | Enrichment analysis of putative targets of Paeonia lactiflora P. for Parkinson's disease. (A) Top 20 Gene Ontology biological processes. (B) Top 20 Kyoto Encyclopedia of Genes and Genomes (KEGG) pathways and Parkinson's disease Pathway. Size of the bubble stands for the number of targets in the pathway and the color stands for $p$ value. The red bubble represents a smaller $p$ value. $(p<0.01)$.

pathway, cholinergic synapse, serotonergic synapse, PI3K-Akt signaling pathway, and apoptosis. Details were listed in Table 2. Moreover, the PD signaling pathway was also observed, with five targets namely ADORA2A, CASP3, CASP9, DRD1, and SLC6A3.

\section{Ingredient-Target-Pathway Network Analysis}

As shown in Figure 3, six core ingredients were obtained, which were kaempferol, $B$-sitosterol, betulinic acid, palbinone, paeoniflorin, and (+)-catechin in turn. Kaempferol was the most important ingredient (Degree $=57, \mathrm{BC}=0.5483$, and $\mathrm{CC}=0.5643$ ), followed by $ß$-sitosterol $(52,0.2646,0.4634)$. In addition, AKT1, mitogen-activated protein kinase 8(MAPK8), BCL2, TNF, and IL-6 were found to be the top five important targets in this network.

\section{DISCUSSION}

Despite substantial advances in the molecular mechanism of PD, conventional clinical trials of single gene and single channel drugs have failed to slow the progression of PD (Olanow et al., 2009; Schapira et al., 2013; Verschuur et al., 2019). The possible reasons are as follows: first, the etiology of $\mathrm{PD}$, involving complex genetic and environmental factors, is difficult to identify, very likely due to highly individualized reasons (Ramsay et al., 2016). Secondly, PD pathology is widely distributed in both central nervous system and peripheral nervous system (Braak et al., 2004), affecting several neurotransmitter systems other than dopaminergic (Sanjari Moghaddam et al., 2017). Therefore, one of the latest trends in the research of disease-modifying drugs is the reasonable combination of multiple targets (Kalia and Lang, 2015; Ke et al., 2016). Coincidentally, TCM exerts its effect through multiple targets and pathways with multiple components, which provides symptomatic relief for PD patients and shows neuroprotective effect on rodents in vivo and in vitro. Extensive use of P. lactiflora in TCM clinics aroused our interest in this herb, and then a network pharmacology approach was adopted to explore its mechanism on a holistic view. In this study, seven active ingredients, six core targets of $P$. lactiflora and related pathways in the treatment of PD were identified. A Schematic diagram of the underlying mechanism is shown in Figure 4.

\section{Paeonia lactiflora may Contribute to the Treatment of Parkinson's Disease by Reducing Neuroinflammation}

Abundant evidence supports that neuroinflammation is an important factor of PD whereby oxidative stress and apoptosis caused by neuroinflammatory response can damage dopaminergic neurons (Hirsch and Hunot, 2009; GuzmanMartinez et al., 2019). Epidemiological investigations also found that non-steroidal anti-inflammatory drugs (NSAIDs) are related to a lower risk of developing PD (Noyce et al., 2012), suggesting that neuroinflammation may promote disease progression. In addition, emerging evidence demonstrates that intestinal inflammation may also be involved in PD pathophysiology through the regulation of the gut-brain axis (Chen et al., 2019). 
TABLE 2 | Kyoto Encyclopedia of Genes and Genomes pathway enrichment of Paeonia lactiflora P. for PD.

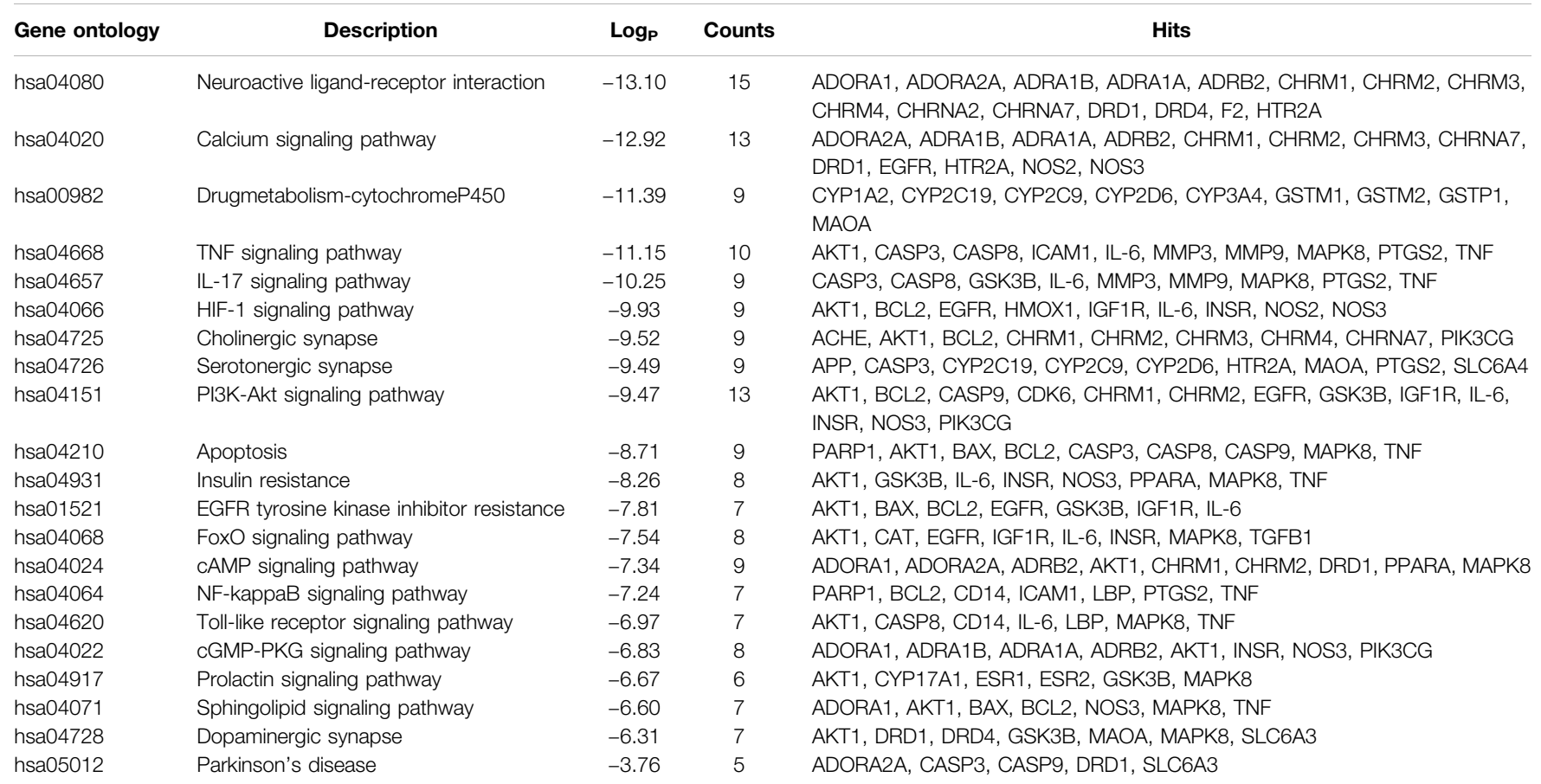

IL-6, Interleukin 6; TNF, Tumor Necrosis Factor; IL-6, Interleukin 6; CASP8, Caspase-8; CASP9, Caspase-9; CASP3, Caspase-3; PTGS2, Cyclooxygenase 2 (COX2); BCL2, Apoptosis regulator BCl-2; AKT1, RAC-alpha serine/threonine-protein kinase (PKB); ADORA2A, Adenosine receptor.

We found that the TNF signaling pathway is a key pathway of $P$. lactiflora for PD. Initiated from a potent pro-inflammatory cytokine TNF, TNF signaling pathway mediates a wide range of cellular processes including inflammation, proliferation, cell migration, apoptosis, and necrosis (McCoy and Tansey, 2008). After TNF binds to TNF Receptor 1 (TNFR1), the adaptor proteins including receptor interacting protein and TNF receptor associated factor 2 (TRAF2) will form a complex (Hsu et al., 1996). This complex can trigger the activation of a series of signaling pathways downstream, including nuclear factor kappa-B (NF- $\mathrm{kB}$ ) signaling pathway, MAPK signaling pathway, and ubiquitin proteasome signaling pathway (Winston et al., 1995). Translocation of NF-кB to the nucleus is a crucial step where cell survival genes, pro-inflammatory cytokines, chemokines, growth factors and TNF $\alpha$ itself were transcribed. Many of these activities participate in inflammation, such as IL-6 production, cyclooxygenase-2 (COX2) synthesis, extracellular matrix remodeling, leukocyte recruitment, (Newton and Dixit, 2012). In addition, autopsy study of PD patients found upregulation of TNFR1 expression on dopaminergic neurons, and activation of central glial cells and peripheral immune cells (Mogi et al., 1996). A recent research showed that the effect of an NF-kB inhibitor $(50 \mathrm{mg} / \mathrm{kg})$ is comparable to levodopa-carbidopa combination in lipopolysaccharide (LPS) induced rats, with a decrease in TNF- $\alpha$ and IL-6 levels (Saini et al., 2020). These findings further confirm that inhibition of TNF signaling pathway might be associated with reducing neuroinflammation in PD. According to the results of I-T-P network analysis, kaempferol and paeoniflorin in $P$. lactiflora may reduce neuroinflammation by inhibiting the expression of TNF and IL-6 in the treatment of PD.

\section{Paeonialactiflora may Contribute to the Treatment of Parkinson's Disease by Inhibiting Apoptosis}

Apoptosis is proved to be a death mode of neurons in substantia nigra in PD patients. Unlike cell necrosis, apoptosis is an active death process mediated by intrinsic or extrinsic signaling pathways, regulated by caspase family, apoptosis regulator (BCL2), family and other genes (Perier et al., 2012).

We found that AKT1, CASP3 and PTGS2 are the key targets of $P$. lactiflora in the treatment of PD, and BCL2 is also among the targets. AKT, also known as protein kinase B, plays a key role in a variety of signaling pathways with more than 100 substrates. AKT phosphorylation induces the binding of accessory proteins necessary for the anti-apoptosis gene BCL2 expression, which in turn inhibits apoptosis by regulating the permeability of the mitochondrial outer membrane (Hers et al., 2011). Another target PTGS2, also known as COX2, is a key prostaglandin synthase induced by inflammation, which is also the target of NSAIDs such as ibuprofen. Generally, PTGS2 is not expressed in dopaminergic neurons, while it is significantly positive in PD patients and mouse models (Teismann et al., 2003). Evidence proves that COX2 inhibition mitigates BCL2, Caspase-9 (CASP9), and Caspase-3 (CASP3) expression through the intrinsic apoptosis 


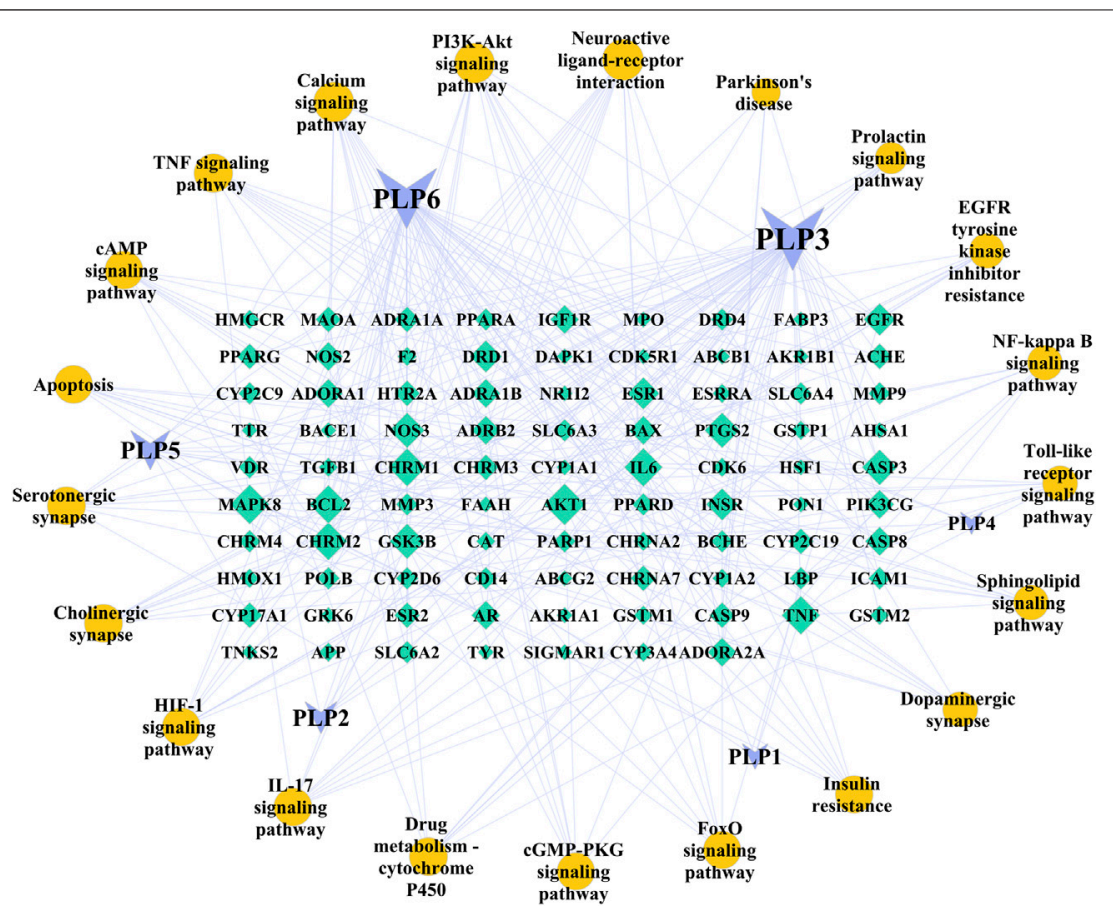

FIGURE 3 | Ingredient-Target-Pathway (I-T-P) network of Paeonia lactiflora P. for Parkinson's disease. The purple inverted triangles represent the ingredients of Paeonia lactiflora P., the green diamonds represent the common targets, and the yellow circles represent the main pathways. Node size is proportional to its degree. The larger the area is, the more important it is in the network. The nodes of ingredients and pathways are arranged clockwise by size. PLP1, paeoniflorin; PLP2, palbinone; PLP3, kaempferol; PLP4, (+)-catechin; PLP5, betulinic acid; PLP6, beta-sitosterol.

pathway (Chauhan et al., 2018). In addition, in the aforementioned inflammatory signaling pathway, TNF/TNFR1 can also bind to FAS-associated death domain protein, activating extrinsic apoptosis pathway, and further leads to apoptosis through the activation of key proteolytic enzymes Caspase-8 (CASP8) and CASP3 (McCoy and Tansey, 2008). According to the results of I-T-P network analysis, kaempferol in $P$. lactiflora may regulate AKT1, CASP3, and BCL2, as well as kaempferol, $B$ sitosterol, and (+)-catechin may regulate PTGS2 to inhibit intrinsic and extrinsic apoptosis in the treatment of PD.

\section{Paeonia lactiflora may Improve the Motor Symptoms and Non-Motor Symptoms of Parkinson's Disease by Regulating Multiple Neurotransmitters level}

According to the classical model of basal ganglion circuit, dyskinesia in PD is caused by unbalanced activity of direct and indirect pathways. The decline of dopamine transmitter released from the substantia nigra to the striatum leads to a decrease in the excitability of the direct pathway (dopamine D1 receptor, DRD1) and the over-activation of the indirect pathway (dopamine D2 receptor, DRD2; adenosine receptor A2a, ADORA2A), which collectively suppress the cortical excitability (McGregor and Nelson, 2019). A2A receptor antagonists is a non-dopamine drug in advanced PD which can shorten the "off" time and improve motor symptoms (Mizuno et al., 2013). AKT1, located downstream of the DRD2, is also involved in the dopamine signaling cascade and affect the expression of dopamine-related behavior in the striatum (Beaulieu et al., 2007). The Sodiumdependent dopamine transporter (SLC6A3) is in charge of dopamine concentration regulation by mediating reuptake on the presynaptic membrane (Habak et al., 2014). I-T-P network analysis showed that $P$. lactiflora may regulate DRD1, ADORA2A, and SLC6A3 in the PD pathway and AKT1 downstream to relieve motor symptoms.

NMS are key determinants of quality of life. However, limited treatments are available (Schapira et al., 2017). Mainstream dopaminergic therapy has no effect on NMS caused by defects in other neurotransmitter pathways, which even aggravates the problem such as constipation (Schaeffer and Berg, 2017). Clinical use of selective serotonin receptor (5-HT) agonists, such as Mosapride, suggests damage to serotonergic nerves (Sakakibara et al., 1996). Selective anticholinergic drugs such as Solifenacin may have an effect on overactive bladder syndrome (Zesiewicz et al., 2015). The enrichment analysis indicates that neuroactive ligand-receptor interaction is the most important pathway of $P$. lactiflora for $\mathrm{PD}$, as well as serotonergic synaptic pathway, cholinergic synaptic pathway, and dopaminergic synaptic pathway also being observed, suggesting that $P$. lactiflora may relieve NMS through the regulation of a variety of neurotransmitter pathways.

Likewise, several studies have clearly demonstrated the neuroprotective effects of $P$. lactiflora components. For example, 


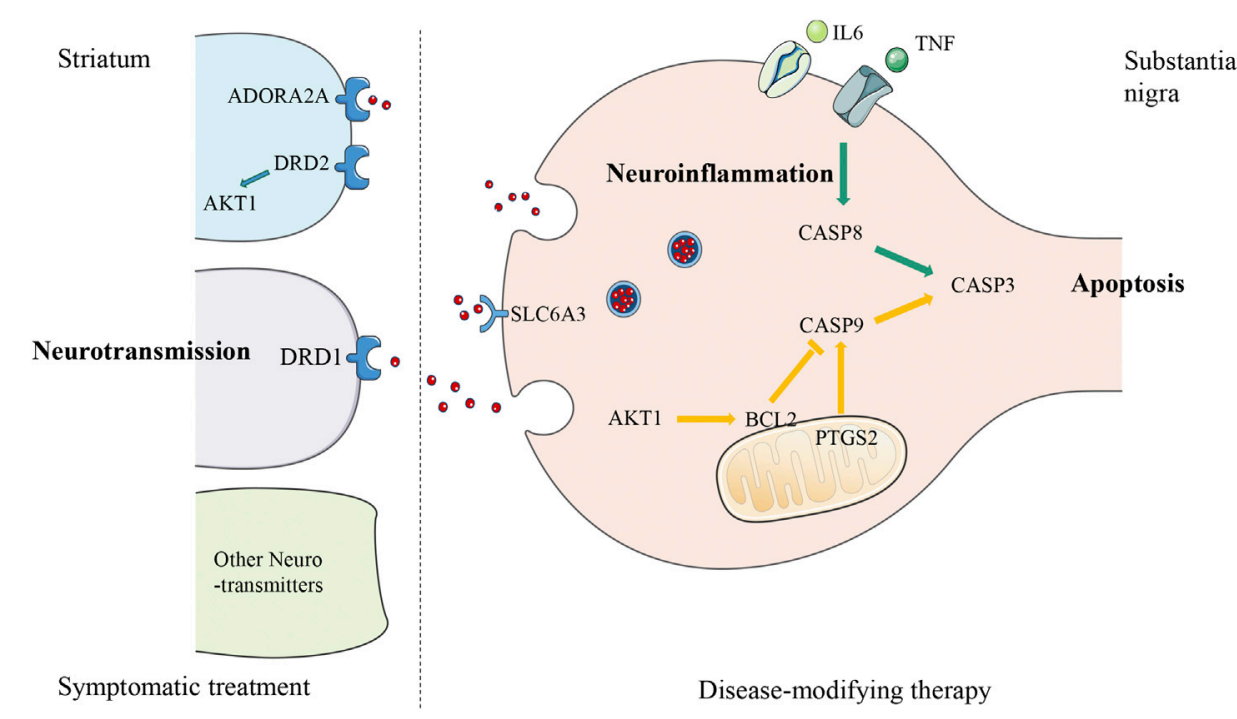

FIGURE 4 | Schematic diagram of the underlying mechanism of Paeonia lactiflora P. in Parkinson's disease. The diagram of a nigro-striatal synapse shows three possible approaches that can be used as therapeutic targets. Parkinson's disease is caused by the early death of dopaminergic neurons in midbrain substantia nigra leading to the reduction of dopamine neurotransmitters released to the striatum, so treatment could be either targeting the degeneration process as disease-modifying therapy (presynaptic, right side of the diagram), or synaptic transmission as symptomatic treatment (postsynaptic, left side of the diagram). The putative targets of Paeonia lactiflora P. are located on three pathways: the green arrows from TNF, IL-6, to CASP8, CASP3 are part of the extrinsic apoptosis pathway induced by neuroinflammation, the yellow arrows from CASP9 to CASP3 with the involvement of AKT1, BCL2, and PTGS2, belong to the intrinsic apoptosis pathway, and blue receptors DRD1, DRD2, ADORA2A, SLC6A3 and AKT1 are related to the regulation of neurotransmitters level. TNF, Tumor Necrosis Factor; IL-6, Interleukin 6; CASP8, Caspase-8; CASP9, Caspase-9; CASP3, Caspase-3; PTGS2, cyclooxygenase 2 (COX2); BCL2, Apoptosis regulator Bcl-2; AKT1, RAC-alpha serine/threonine-protein kinase (PKB); ADORA2A, Adenosine receptor A2a; DRD1, Dopamine D1 receptor; DRD2, Dopamine D2 receptor; SLC6A3, Sodium-dependent dopamine transporter.

kaempferol inhibits the activation of NLRP3 inflammasome through the synergistic effect of ubiquitin and autophagy, thus promotes the survival of dopaminergic neurons in PD models induced by LPS and SNCA (Han et al., 2019). Kaempferol can also maintain the stability of blood-brain barrier and down-regulate HMGB1/TLR4 pathway to reduce striatal injury in mice (Yang et al., 2019). A number of studies showed that paeoniflorin protects PC12 cells against $\mathrm{MPP}+$, acid, and glutamate induced neuron injury via regulating autophagy, apoptosis, and mitochondrial membrane potential (Cao et al., 2010; Sun et al., 2012; Zheng et al., 2016). It also reduces dopaminergic neurodegeneration in MPTP model by inhibition of neuroinflammation and suppression of the accelerated dopamine catabolism via Amine oxidase B (MAOB) inhibition (Liu et al., 2006; Zheng et al., 2017). B-sitosterol enhances membrane potential and ATP content of mitochondria which is expected to benefit AD (Shi et al., 2013). Betulinic acid, also known as mairin, induces apoptosis in cells through the mitochondrial pathway mediated by ROS (Wang et al., 2017).

Besides anti-parkinsonian property, P. lactiflora also exhibits neuroprotective potential in other neurodegenerative diseases. Acetylcholinesterase (AChE) inhibitor is a first-line treatment for $\mathrm{AD}$ dementia, and possibly useful for PD dementia (Seppi et al., 2019). Lin compared the AChE inhibitory activities among 26 herbs in vitro, while ethanol extracts of $P$. lactiflora roots showed the second strongest inhibition $\left(\mathrm{IC}_{50}=8 \mu \mathrm{g} / \mathrm{ml}\right)$ in a dosedependent manner (Lin et al., 2008). Albiflorin, one of the core components of $P$. lactiflora, ameliorates memory deficits and reduces brain amyloid- $\beta$ deposition in APP/PS1 mice (Xu et al., 2019). Above mentioned TGP can reduce the severity and progression of experimental autoimmune encephalomyelitis, a multiple sclerosis model in mice, by attenuating inflammation response (Huang et al., 2015).

Among more than 50 types of plants against MPTP-induced neurotoxicity in a recent review, antioxidant, antiapoptotic, and autophagy enhancement are the most studied mechanisms (Abushouk et al., 2017). P. lactiflora and Mucuna pruriens seeds have similar mechanism in ameliorating neuroinflammation and apoptosis, while the latter contains natural levodopa used as a dopamine supplement in India (Lieu et al., 2012; Rai et al., 2017). Both $P$. lactiflora and Sophora Tomentosa exert antioxidant properties, which might be associated with their common component, catechin (Chang et al., 2019). Although P. lactiflora is a major component in several formulas reported to be neuroprotective in vivo (Ahn et al., 2019; Tang et al., 2020), this is the first time to explore its mechanism alone from a systematic level, which may benefit further drug manufacturing.

Our study has several limitations. First, since the network pharmacology approach is performed by target prediction based on existing studies, the unique ingredients of $P$. lactiflora like paeoniflorin is less recognized compared to common ingredients like kaempferol, which may affect the importance of the components in the target network. Secondly, further experiments should verify the conclusion drawn in this study, including the pathways, neurotransmitters level, and neuron degeneration. Another interesting question to answer is whether the components play a role through the peripheral or 
central nervous system. Future research aimed toward novel drug development must consider safety as well as efficacy of the components; and down the line, do the results obtained in cellular or animal models translate to the bedside?

\section{CONCLUSION}

Taken together, $P$. lactiflora may retard neurodegeneration by reducing neuroinflammation, inhibiting intrinsic and extrinsic apoptosis, and improve motor and NMS by regulating the level of neurotransmitters. Our study has revealed the underlying multicomponent, multitarget, and multipathway mechanism of P. lactiflora in the treatment of $\mathrm{PD}$, which may contribute to novel drug development for PD.

\section{DATA AVAILABILITY STATEMENT}

The raw data supporting the conclusions of this article will be made available by the authors, without undue reservation.

\section{REFERENCES}

Abushouk, A. I., Negida, A., Ahmed, H., and Abdel-Daim, M. M. (2017). Neuroprotective mechanisms of plant extracts against MPTP induced neurotoxicity: future applications in Parkinson's disease. Biomed. Pharmacother. 85, 635-645. doi:10.1016/j.biopha.2016.11.074

Ahn, S., Liu, Q. F., Jang, J. H., Park, J., Jeong, H. J., Kim, Y., et al. (2019). Gamichunggan formula prevents motor dysfunction in MPTP/p-Induced and A53T a-synuclein overexpressed Parkinson's disease mouse model though DJ-1 and BDNF expression. Front. Aging Neurosci. 11, 230. doi:10.3389/fnagi.2019.00230

Amberger, J. S., Bocchini, C. A., Schiettecatte, F., Scott, A. F., and Hamosh, A. (2015). OMIM.org: online Mendelian Inheritance in Man $\left(\mathrm{OMIM}^{\circledR}\right)$, an online catalog of human genes and genetic disorders. Nucleic Acids Res. 43, D789-D798. doi:10.1093/nar/gku1205

Beaulieu, J. M., Gainetdinov, R. R., and Caron, M. G. (2007). The Akt-GSK-3 signaling cascade in the actions of dopamine. Trends Pharmacolo. Sci. 28 (4), 166-172. doi:10.1016/j.tips.2007.02.006

Braak, H., Ghebremedhin, E., Rüb, U., Bratzke, H., and Del Tredici, K. (2004). Stages in the development of Parkinson's disease-related pathology. Cell Tissue Res. 318 (1), 121-134. doi:10.1007/s00441-004-0956-9

Cao, B.-Y., Yang, Y.-P., Luo, W.-F., Mao, C.-J., Han, R., Sun, X., et al. (2010). Paeoniflorin, a potent natural compound, protects PC12 cells from MPP+ and acidic damage via autophagic pathway. J. Ethnopharmacol. 131 (1), 122-129. doi:10.1016/j.jep.2010.06.009.

Chang, H. C., Liu, K. F., Teng, C. J., Lai, S. C., Yang, S. E., Ching, H., et al. (2019). Sophora Tomentosa extract prevents MPTP-induced parkinsonism in C57bl/6 mice via the inhibition of GSK-3 $\beta$ phosphorylation and oxidative stress. Nutrients 11 (2), 252. doi:10.3390/nu11020252

Chauhan, A. K., Mittra, N., Patel, D. K., and Singh, C. (2018). Cyclooxygenase-2 directs microglial activation-mediated inflammation and oxidative stress leading to intrinsic apoptosis in Zn-induced parkinsonism. Mol. Neurobiol. 55 (3), 2162-2173. doi:10.1007/s12035-017-0455-0

Chen, Q. Q., Haikal, C., Li, W., and Li, J. Y.(2019). Gut inflammation in association with pathogenesis of Parkinson's disease. Front. Mol. Neurosci. 12, 218. doi:10. 3389/fnmol.2019.00218

de Bie, R. M. A., Clarke, C. E., Espay, A. J., Fox, S. H., and Lang, A. E. (2020). Initiation of pharmacological therapy in Parkinson's disease: when, why, and how. Lancet Neurol. 19 (5), 452-461. doi:10.1016/s1474-4422(20)30036-3

Dorsey, E. R., and Bloem, B. R.(2018). The Parkinson pandemic-A call to action. JAMA Neurol. 75 (1), 9-10. doi:10.1001/jamaneurol.2017.3299

\section{AUTHOR CONTRIBUTIONS}

WD, YZ conceived this work and drafted the manuscript, XL, SW collected the data, and PL assisted in the revision of the manuscript. All the authors revised and approved the manuscript.

\section{FUNDING}

This research was supported by the National TCM Leading Personnel Support Program (NATCM Personnel and Education Department [2018] No.12).

\section{ACKNOWLEDGMENTS}

The authors thank Dan Wenchao for assistance with software support, Ji Shaozhen and Liu Zeyu for their useful comments, Ma Boyuan for proofreading the manuscript, and all members of the Zhang's team in clinic for continuous observation on cases. They thank the editors and reviewers for their professional suggestions to improve this work.

Elkouzi, A., Vedam-Mai, V., Eisinger, R. S., and Okun, M. S. (2019). Emerging therapies in Parkinson disease - repurposed drugs and new approaches. Nat. Rev. Neurol. 15 (4), 204-223. doi:10.1038/s41582-019-0155-7

Feng, Z., Zhang, B. Q., Zhu, Y. M., Yu, B. B., Fu, L., Zhou, . L., et al. (2019). The effectiveness and safety of total glucosides of paeony in primary Sjögren's syndrome: a systematic review and meta-analysis. Front. Pharmacol. 10, 550. doi:10.3389/fphar.2019.00550

Gfeller, D., Grosdidier, A., Wirth, M., Daina, A., Michielin, O., and Zoete, V. (2014). SwissTargetPrediction: a web server for target prediction of bioactive small molecules. Nucleic Acids Res. 42, W32-W38. doi:10.1093/nar/gku293

Guzman-Martinez, L., Maccioni, R. B., Andrade, V., Navarrete, L. P., Pastor, M. G., and Ramos-Escobar, N. (2019). Neuroinflammation as a common feature of neurodegenerative disorders. Front. Pharmacol. 10, 1008. doi:10.3389/fphar. 2019.01008

Habak, C., Noreau, A., Nagano-Saito, A., Mejía-Constaín, B., Degroot, C., Strafella, A. P., et al. (2014). Dopamine transporter SLC6A3 genotype affects corticostriatal activity of set-shifts in Parkinson's disease. Brain 137 (Pt 11), 3025-3035. doi:10.1093/brain/awu251

Han, X., Sun, S., Sun, Y., Song, Q., Zhu, J., Song, N., et al. (2019). Small moleculedriven NLRP3 inflammation inhibition via interplay between ubiquitination and autophagy: implications for Parkinson disease. Autophagy 15 (11), 1860-1881. doi:10.1080/15548627.2019.1596481

Hers, I., Vincent, E. E., and Tavaré, J. M. (2011). Akt signalling in health and disease. Cell Signal. 23 (10), 1515-1527. doi:10.1016/j.cellsig.2011.05.004

Hirsch, E. C., and Hunot, S. (2009). Neuroinflammation in Parkinson's disease: a target for neuroprotection? Lancet Neurol. 8 (4), 382-397. doi:10.1016/s14744422(09)70062-6

Hopkins, A. L. (2007). Network pharmacology. Nat. Biotechnol. 25 (10), 1110-1111. doi:10.1038/nbt1007-1110

Hsu, H., Huang, J., Shu, H. B., Baichwal, V., and Goeddel, D. V. (1996). TNFdependent recruitment of the protein kinase RIP to the TNF receptor-1 signaling complex. Immunity 4 (4), 387-396. doi:10.1016/s1074-7613(00) 80252-6

Huang, Q., Ma, X., Zhu, D. L., Chen, L., Jiang, Y., Zhou, L., et al. (2015). Total glucosides of peony attenuates experimental autoimmune encephalomyelitis in C57BL/6 mice. J. Neuroimmunol. 284, 67-73. doi:10.1016/j.jneuroim.2015.05.009

Kadota, S., Terashima, S., Basnet, P., Kikuchi, T., and Namba, T. (1993). Palbinone, a novel terpenoid from Paeonia albiflora; potent inhibitory activity on 3 alphahydroxysteroid dehydrogenase. Chem. Pharmaceut. Bull. 41 (3), 487-490. doi: $10.1248 /$ cpb. 41.487 
Kalia, L., and Lang, A. (2015). Parkinson's disease. Lancet (London, England) 386 (9996), 896-912. doi:10.1016/s0140-6736(14)61393-3

Ke, Z., Zhang, X., Cao, Z., Ding, Y., Li, N., Cao, L., et al. (2016). Drug discovery of neurodegenerative disease through network pharmacology approach in herbs. Biomed. Pharmacother 78, 272-279. doi:10.1016/j.biopha.2016.01.021

Kibble, M., Saarinen, N., Tang, J., Wennerberg, K., Mäkelä, S., and Aittokallio, T. (2015). Network pharmacology applications to map the unexplored target space and therapeutic potential of natural products. Nat. Prod. Rep. 32 (8), 1249-1266. doi:10.1039/c5np00005j

Klingelhoefer, L., and Reichmann, H. (2015). Pathogenesis of Parkinson disease-the gut-brain axis and environmental factors. Nat. Rev. Neurol. 11 (11), 625-636. doi:10.1038/nrneurol.2015.197

Li, L., Qiu, H., Liu, M., and Cai, Y. (2020). A network pharmacology-based study of the molecular mechanisms of shaoyao-Gancao decoction in treating Parkinson's disease. Interdiscip. Sci. 12, 131-144. doi:10.1007/s12539-02000359-7

Lieu, C. A., Venkiteswaran, K., Gilmour, T. P., Rao, A. N., Petticoffer, A. C., Gilbert, E. V., et al. (2012). The antiparkinsonian and antidyskinetic mechanisms of Mucuna pruriens in the MPTP-treated nonhuman primate. Evid. Based Compl. Alternat. Med. 2012, 840247. doi:10.1155/2012/840247

Lin, H. Q., Ho, M. T., Lau, L. S., Wong, K. K., Shaw, P. C., and Wan, D. C. (2008). Anti-acetylcholinesterase activities of traditional Chinese medicine for treating Alzheimer's disease. Chem. Biol. Interact. 175 (1-3), 352-354. doi:10.1016/j.cbi. 2008.05.030

Liu, H. Q., Zhang, W. Y., Luo, X. T., Ye, Y., and Zhu, X. Z. (2006). Paeoniflorin attenuates neuroinflammation and dopaminergic neurodegeneration in the MPTP model of Parkinson's disease by activation of adenosine A1 receptor. $B r$. J. Pharmacol. 148 (3), 314-325. doi:10.1038/sj.bjp.0706732

Liu, J., Chen, L., Fan, C. R., Li, H., Huang, M. Q., Xiang, Q., et al. (2015). Qualitative and quantitative analysis of major constituents of Paeoniae Radix alba and Paeoniae Radix rubra by HPLC-DAD-Q-TOF-MS/MS. Chin. J. Chin. Mat. Med. 40 (9), 1762-1770. doi:10.4268/cjcmm20150926

Luo, J., Jin, D. E., Yang, G. Y., Zhang, Y. Z., Wang, J. M., Kong, W. P., et al. (2017). Total glucosides of paeony for rheumatoid arthritis: a systematic review of randomized controlled trials. Compl. Ther. Med. 34, 46-56. doi:10.1016/j.ctim. 2017.07.010

McCoy, M. K., and Tansey, M. G. (2008). TNF signaling inhibition in the CNS: implications for normal brain function and neurodegenerative disease. J. Neuroinflammation 5, 45. doi:10.1186/1742-2094-5-45

McGregor, M. M., and Nelson, A. B. (2019). Circuit mechanisms of Parkinson's disease. Neuron 101 (6), 1042-1056. doi:10.1016/j.neuron.2019.03.004

Mizuno, Y., and Kondo, T., and Japanese Istradefylline Study Group (2013). Adenosine A2A receptor antagonist istradefylline reduces daily OFF time in Parkinson's disease. Mov. Disord. 28 (8), 1138-1141. doi:10.1002/mds.25418

Mogi, M., Togari, A., Kondo, T., Mizuno, Y., Komure, O., Kuno, S., et al. (2000). Caspase activities and tumor necrosis factor receptor R1 (p55) level are elevated in the substantia nigra from parkinsonian brain. J. Neur. Transm. Vienna 107 (3), 335-341. doi:10.1007/s007020050028

National Institute for Health and Care Excellence (2017). Parkinson's disease in adults: diagnosis and management. London, UK: National Institute for Health and Care Excellence (UK) Copyright $\odot$ NICE.

National Pharmacopoeia Commission (2010). Pharmacopoeia of the People's Republic of China. Beijing, China: China Medical Science Press (In Chinese).

Newton, K., and Dixit, V. M. (2012). Signaling in innate immunity and inflammation. Cold Spring Har. Perspe. Biolo. 4 (3). doi:10.1101/cshperspect.a006049

Noyce, A. J., Bestwick, J. P., Silveira-Moriyama, L., Hawkes, C. H., Giovannoni, G., et al. (2012). Meta-analysis of early nonmotor features and risk factors for Parkinson disease. Ann. Neurol. 72 (6), 893-901. doi:10.1002/ana.23687

Olanow, C. W., Rascol, O., Hauser, R., Feigin, P. D., Jankovic, J., Lang, A., et al. (2009). A double-blind, delayed-start trial of rasagiline in Parkinson's disease. N. Engl. J. Med. 361 (13), 1268-1278. doi:10.1056/NEJMoa0809335

Perier, C., Bové, J., and Vila, M. (2012). Mitochondria and programmed cell death in Parkinson's disease: apoptosis and beyond. Antioxid. Redox Signal. 16 (9), 883-895. doi:10.1089/ars.2011.4074

Piñero, J., Bravo, À, Queralt-Rosinach, N., Gutiérrez-Sacristán, A., Deu-Pons, J., Centeno, E., et al. (2017). DisGeNET: a comprehensive platform integrating information on human disease-associated genes and variants. Nucleic Acids Res. 45 (D1), D833-D839. doi:10.1093/nar/gkw943
Rai, S. N., Birla, H., Singh, S. S., Zahra, W., Patil, R. R., Jadhav, J. P., et al. (2017). Mucuna pruriens protects against MPTP intoxicated neuroinflammation in Parkinson's disease through NF-kB/pAKT signaling pathways. Front. Aging Neurosci. 9, 421. doi:10.3389/fnagi.2017.00421

Ramsay, R. R., Majekova, M., Medina, M., and Valoti, M. (2016). Key targets for multi-target ligands designed to combat neurodegeneration. Front. Neurosci. 10, 375. doi:10.3389/fnins.2016.00375

Ru, J., Li, P., Wang, J., Zhou, W., Li, B., Huang, C., et al. (2014). TCMSP: a database of systems pharmacology for drug discovery from herbal medicines. J. Cheminf. 6, 13. doi:10.1186/1758-2946-6-13

Saini, N., Akhtar, A., Chauhan, M., Dhingra, N., and Pilkhwal Sah, S. (2020). Protective effect of Indole-3-carbinol, an NF-kB inhibitor in experimental paradigm of Parkinson's disease: in silico and in vivo studies. Brain Behav. Immun. 90, 108-137. doi:10.1016/j.bbi.2020.08.001

Sakakibara, R., Uchiyama, T., Yamanishi, T., Shirai, K., and Hattori, T. (2008). Bladder and bowel dysfunction in Parkinson's disease. J. Neur. Transm. Vienna 115 (3), 443-460. doi:10.1007/s00702-007-0855-9

Sanjari Moghaddam, H., Zare-Shahabadi, A., Rahmani, F., and Rezaei, N. (2017). Neurotransmission systems in Parkinson's disease. Rev. Neurosci. 28 (5), 509-536. doi:10.1515/revneuro-2016-0068

Schaeffer, E., and Berg, D. (2017). Dopaminergic therapies for non-motor symptoms in Parkinson's disease. CNS Drugs 31 (7), 551-570. doi:10.1007/ s40263-017-0450-z

Schapira, A. H., McDermott, M. P., Barone, P., Comella, C. L., Albrecht, S., Hsu, H. H., et al. (2013). Pramipexole in patients with early Parkinson's disease (PROUD): a randomised delayed-start trial. Lancet Neurol. 12 (8), 747-755. doi:10.1016/s1474-4422(13)70117-0

Schapira, A. H. V., Chaudhuri, K. R., and Jenner, P. (2017). Non-motor features of Parkinson disease. Nat. Rev. Neurosci. 18 (7), 435-450. doi:10.1038/nrn.2017.

Seppi, K., Ray Chaudhuri, K., Coelho, M., Fox, S. H., Katzenschlager, R., Perez Lloret, S., et al. (2019). Update on treatments for nonmotor symptoms of Parkinson's disease-an evidence-based medicine review. Mov. Disord. 34 (2), 180-198. doi:10.1002/mds.27602

Shannon, P., Markiel, A., Ozier, O., Baliga, N. S., Wang, J. T., Ramage, D., et al. (2003). Cytoscape: a software environment for integrated models of biomolecular interaction networks. Genome Res. 13 (11), 2498-2504. doi:10.1101/gr.1239303

Shi, C., Wu, F., and Xu, J. (2013). Incorporation of $\beta$-sitosterol into mitochondrial membrane enhances mitochondrial function by promoting inner mitochondrial membrane fluidity. J. Bioenerg. Biomembr. 45 (3), 301-305. doi:10.1007/s10863-012-9495-3

Shu, X., Duan, W., Liu, F., Shi, X., Geng, Y., Wang, X., et al. (2014). Preparative separation of polyphenols from the flowers of Paeonia lactiflora Pall. by highspeed counter-current chromatography. J. Chromatogr. B Analyt. Technol. Biomed. Life Sci. 947-948, 62-7. doi:10.1016/j.jchromb.2013.12.004

Sun, R., Wang, K., Wu, D., Li, X., and Ou, Y. (2012). Protective effect of paeoniflorin against glutamate-induced neurotoxicity in PC12 cells via Bcl-2/Bax signal pathway. Folia Neuropathol. 50 (3), 270-276. doi:10.5114/fn.2012.30527

Sun, Z. Y., and Peng, W. (2017). Regularity of herbal medicine in treating Parkinson's disease based on data mining. Pract. J. Card. Cerebr. Pneum. Vascul. Dis. 25 (5), 83-86. doi:10. 3969/j. issn. 1008-5971. 2017. 05. 022.

Szklarczyk, D., Morris, J. H., Cook, H., Kuhn, M., Wyder, S., Simonovic, M., et al. (2017). The STRING database in 2017: quality-controlled protein-protein association networks, made broadly accessible. Nucleic Acids Res. 45 (D1), D362-D368. doi:10.1093/nar/gkw937

Tan, Y. Q., Chen, H. W., Li, J., and Wu, Q. J. (2020). Efficacy, chemical constituents, and pharmacological actions of Radix Paeoniae rubra and Radix Paeoniae Alba. Front. Pharmacol. 11, 1054. doi:10.3389/fphar.2020.01054

Tang, L., Chen, C., Xia, B., Wu, W., Wei, R., Zhu, G., et al. (2020). Effect of wenshen-yanggan decoction on movement disorder and substantia nigra dopaminergic neurons in mice with chronic Parkinson's disease. Evid Based Compl. Alternat. Med. 2020, 9838295. doi:10.1155/2020/9838295

Teismann, P., Vila, M., Choi, D. K., Tieu, K., Wu, D. C., Jackson-Lewis, V., et al. (2003). COX-2 and neurodegeneration in Parkinson's disease. Ann. N. Y. Acad. Sci. 991, 272-277. doi:10.1111/j.1749-6632.2003.tb07482.x

UniProt Consortium (2019). UniProt: a worldwide hub of protein knowledge. Nucleic Acids Res. 47 (D1), D506-D515. doi:10.1093/nar/gky1049

Verschuur, C. V. M., Suwijn, S. R., Boel, J. A., Post, B., Bloem, B. R., van Hilten, J. J., et al. (2019). Randomized delayed-start trial of levodopa in Parkinson's disease. N. Engl. J. Med. 380 (4), 315-324. doi:10.1056/NEJMoa1809983 
Wang, J., and Urban, L. (2004). The impact of early ADME profiling on drug discovery and development strategy. Drug Discov. World 5, 73-86.

Wang, X., Lu, X., Zhu, R., Zhang, K., Li, S., Chen, Z., et al. (2017). Betulinic acid induces apoptosis in differentiated PC12 cells via ROS-mediated mitochondrial pathway. Neurochem. Res. 42 (4), 1130-1140. doi:10.1007/s11064-016-2147-y

WHO (2019). WHO global report on traditional and complementary medicine 2019. Geneva, Switzerland: World Health Organization,

Winston, B. W., Lange-Carter, C. A., Gardner, A. M., Johnson, G. L., and Riches, D. W. (1995). Tumor necrosis factor alpha rapidly activates the mitogen-activated protein kinase (MAPK) cascade in a MAPK kinase kinase-dependent, c-Raf-1independent fashion in mouse macrophages. Proc. Natl. Acad. Sci. U.S.A. 92 (5), 1614-1618. doi:10.1073/pnas.92.5.1614

Wishart, D. S., Feunang, Y. D., Guo, A. C., Lo, E. J., Marcu, A., Grant, J. R., et al. (2018). DrugBank 5.0: a major update to the DrugBank database for 2018. Nucleic Acids Res. 46 (D1), D1074-d82. doi:10.1093/nar/gkx1037

Wu, L. F., Wang, X. Q., Chen, X. M., Duan, T. H., Liu, S. Y., Peng, Y. Z., et al. (2020). Chemical constituents and pharmacological activities of Radix Paeoniae Alba: research advances. J. Int. Pharm. Res. 47 (3), 175-187. doi:10.13220/j.cnki.jipr. 2020.03.003.

Xu, Y. J., Mei, Y., Shi, X. Q., Zhang, Y. F., Wang, X. Y., Guan, L., et al. (2019). Albiflorin ameliorates memory deficits in APP/PS1 transgenic mice via ameliorating mitochondrial dysfunction. Brain Res. 1719, 113-123. doi:10. 1016/j.brainres.2019.05.037

Yan, J. M. (2005). Supplementary translation of prescriptions for fifty-two aliments. Beijing, China: Traditional Chinese Medicine Publishing House, 139.

Yang, Y. L., Cheng, X., Li, W. H., Liu, M., Wang, Y. H., and Du, G. H. (2019). Kaempferol attenuates LPS-induced striatum injury in mice involving antineuroinflammation, maintaining $\mathrm{BBB}$ integrity, and down-regulating the HMGB1/TLR4 pathway. Int. J. Mol. Sci. 20 (3), 491. doi:10.3390/ijms20030491

You, S. (2009). Practical traditional Chinese internal medicine. Nanjing, China: Jiangsu Science and Technology Publishing House.

Yu, J., Elix, J. A., and Iskander, M. N. (1990). Lactiflorin, a monoterpene glycoside from paeony root. Phytochemistry 29 (12), 3859-3863. doi:10.1016/00319422(90)85347-I
Zesiewicz, T. A., Evatt, M., Vaughan, C. P., Jahan, I., Singer, C., Ordorica, R., et al. (2015). Randomized, controlled pilot trial of solifenacin succinate for overactive bladder in Parkinson's disease. Park. Relat. Disord. 21 (5), 514-520. doi:10. 1016/j.parkreldis.2015.02.025

Zhang, L., and Wei, W. (2020). Anti-inflammatory and immunoregulatory effects of paeoniflorin and total glucosides of paeony. Pharmacol. Ther. 207, 107452. doi:10.1016/j.pharmthera.2019.107452

Zheng, M., Liu, C., Fan, Y., Shi, D., and Jian, W. (2019). Total glucosides of paeony (TGP) extracted from Radix Paeoniae Alba exerts neuroprotective effects in MPTP-induced experimental parkinsonism by regulating the cAMP/PKA/ CREB signaling pathway. J. Ethnopharmacol. 245, 112182. doi:10.1016/j.jep. 2019.112182

Zheng, M., Liu, C., Fan, Y., Shi, D., and Zhang, Y. (2016). Protective effects of paeoniflorin against MPP(+)-induced neurotoxicity in PC12 cells. Neurochem. Res. 41 (6), 1323-1334. doi:10.1007/s11064-016-1834-Z

Zheng, M., Liu, C., Fan, Y., Yan, P., Shi, D., and Zhang, Y. (2017). Neuroprotection by Paeoniflorin in the MPTP mouse model of Parkinson's disease. Neuropharmacology 116, 412-420. doi:10.1016/j.neuropharm.2017.01.009

Zhou, Y., Zhou, B., Pache, L., Chang, M., Khodabakhshi, A. H., Tanaseichuk, T., et al. (2019). Metascape provides a biologist-oriented resource for the analysis of systems-level datasets. Nat. Commun. 10 (1), 1523. doi:10.1038/s41467-01909234-6

Conflict of Interest: The authors declare that the research was conducted in the absence of any commercial or financial relationships that could be construed as a potential conflict of interest.

Copyright $\odot 2020 \mathrm{Du}$, Liang, Wang, Lee and Zhang. This is an open-access article distributed under the terms of the Creative Commons Attribution License (CC BY). The use, distribution or reproduction in other forums is permitted, provided the original author(s) and the copyright owner(s) are credited and that the original publication in this journal is cited, in accordance with accepted academic practice. No use, distribution or reproduction is permitted which does not comply with these terms. 